\title{
Die Bedeutung schulischer Kontexteffekte und adaptiver Lehrkompetenz für das selbstregulierte Lernen
}

\section{Christian Brühwiler}

Im Hinblick auf ein selbstverantwortliches Weiterlernen nach der Schulzeit ist die Fähigkeit zentral, den eigenen Lernprozess erfolgreich zu steuern. Selbstreguliertes Lernen (SRL) ist zugleich Ziel und Bedingung schulischer Lernprozesse. Der vorliegende Beitrag analysiert zunächst anhand der Daten von PISA 2003 die Bedeutung des Schultyps und der Leistungsstärke von Schulen für verschiedene Aspekte des SRL. Die Ergebnisse zeigen, dass sich auch für die Schweiz ein "big-fish-little-pond-effect» (BFLPE; Marsh, 1987) nachweisen lässt. Schülerinnen und Schüler verfügen demnach in leistungsstarken Schulen über ein geringeres mathematisches Selbstkonzept als Schülerinnen und Schüler mit gleichen individuellen Mathematikleistungen in leistungsschwächeren Schulen. Vor dem Hintergrund der hohen Bedeutung des SRL für schulischen Lernerfolg wird in einer zweiten Studie der Frage nachgegangen, welchen Beitrag Lehrpersonen zur Förderung des SRL leisten können. Die empirische Grundlage für die Analysen bilden 49 Lehrpersonen und ihre Klassen, die an der Nationalfonds-Studie "Adaptive Lehrkompetenz»" ${ }^{1}$ teilgenommen haben. Als adaptive Lehrkompetenz wird die Fähigkeit von Lehrpersonen bezeichnet, Unterrichtsvorbereitung und-handeln so auf die individuellen Voraussetzungen der Lernenden auszurichten, dass möglichst günstige Bedingungen für verstehendes Lernen entstehen. Die Ergebnisse verweisen darauf, dass zur Förderung bereichsspezifischer Interessen und Selbstkonzepte diagnostische und didaktische Kompetenzen der Lehrpersonen ausschlaggebend sind, sofern sie sich direkt aufs Unterrichtsgeschehen beziehen.

\section{Einleitung}

Will die Schule auf künftige Anforderungen im Erwachsenenleben vorbereiten, muss sie neben fachlichen Kompetenzen auch überfachliche Lernfähigkeiten vermitteln. Schülerinnen und Schüler sollen befähigt werden, selbstständig Lernprozesse $\mathrm{zu}$ initiieren, diese zu steuern und aufrecht zu erhalten. Solche Lernfähigkeiten werden unter dem Begriff selbstreguliertes Lernen (SRL) zusammengefasst (Artelt, 2000; Boekaerts, 1999) und besitzen eine doppelte 
Funktion: Einerseits stellen sie die Grundlagen für ein selbstverantwortliches Weiterlernen im Erwachsenenleben dar und unterstützen damit lebenslanges Lernen. Andererseits verfügen selbstreguliert Lernende über gute Voraussetzungen beim Erwerb fachlicher Kompetenzen. Zahlreiche Forschungsergebnisse konnten solche positiven Effekte lernprozessnaher Faktoren auf die schulische Leistung nachweisen (z.B. Artelt, 2000; Artelt, Baumert, Julius-McElvany \& Peschar, 2003). Auch die bisherigen PISA-Ergebnisse haben bestätigt, dass Jugendliche mit hohen fachlichen Kompetenzen über günstigere individuelle Voraussetzungen zur aktiven Steuerung von Lernprozessen verfügen (Artelt et al., 2003; Zutavern \& Brühwiler, 2002). Die Schweizer Analysen zu PISA 2003 erbrachten zudem Hinweise darauf, dass die Ausprägung verschiedener Merkmale des SRL und deren Beziehungen zur Mathematikleistung auch vom besuchten Schultyp abhängen (Brühwiler \& Biedermann, 2005). Ergänzend stellt sich die Frage, ob die Leistungsstärke der Schulen innerhalb der Schultypen für gewisse Merkmale des SRL bedeutsam ist. Vor dem Hintergrund der hohen Bedeutung des SRL drängt sich zudem die Frage nach der Förderung selbstregulierten Lernens auf, insbesondere welchen Beitrag Lehrpersonen leisten können.

Der vorliegende Artikel greift die Befunde aus PISA 2003 zum SRL auf und vertieft sie unter dem Gesichtspunkt des besuchten Schultyps und des Leistungsniveaus der Schulen. Im zweiten Teil des Artikels wird anhand von Daten aus dem Nationalfonds-Projekt «Adaptive Lehrkompetenz» (Beck et al., 2006) untersucht, welche Bedeutung den Kompetenzen von Lehrpersonen bei der Förderung verschiedener Aspekte des SRL zukommt.

\section{Theoretischer Hintergrund}

Als konzeptuelle Grundlage dieses Artikels dient ein theoretisches Rahmenmodell, das die Verbindung zwischen den Kompetenzen der Lehrpersonen und den über Unterrichtsprozesse und SRL vermittelten fachlichen Leistungen darstellt (Abbildung 1). Das Rahmenmodell veranschaulicht in vereinfachter Weise das komplexe Bedingungsgefüge schulischer Lernprozesse sowie die Doppelfunktion des SRL als Ziel und Bedingung schulischen Lernens. Es stützt sich teilweise auf das Angebots-Nutzungs-Modell zur Wirkungsweise des Unterrichts von Helmke (2003) und verbindet es mit Elementen aus den beiden Studien PISA und «Adaptive Lehrkompetenz».

Das Modell beruht auf sechs miteinander verbundenen Bereichen, die sich wechselseitig beeinflussen. Als Zielkriterien des schulischen Lernens werden nicht nur die fachlichen Kompetenzen, sondern auch Fähigkeiten des SRL betrachtet. Im Modell sind die bei PISA 2003 erfassten Kompetenzbereiche Mathematik, Naturwissenschaften, Lesen und Problemlösen aufgeführt. Der Doppelpfeil zwischen dem SRL und den fachlichen Kompetenzen verweist auf den wechselseitigen Zusammenhang. Daraus wird auch die zweifache Funktion des 
SRL ersichtlich: Es ist sowohl Bedingung für schulischen Lernerfolg als auch im Sinne eigenständiger, fächerübergreifender Kompetenzen - Unterrichtsziel selbst.

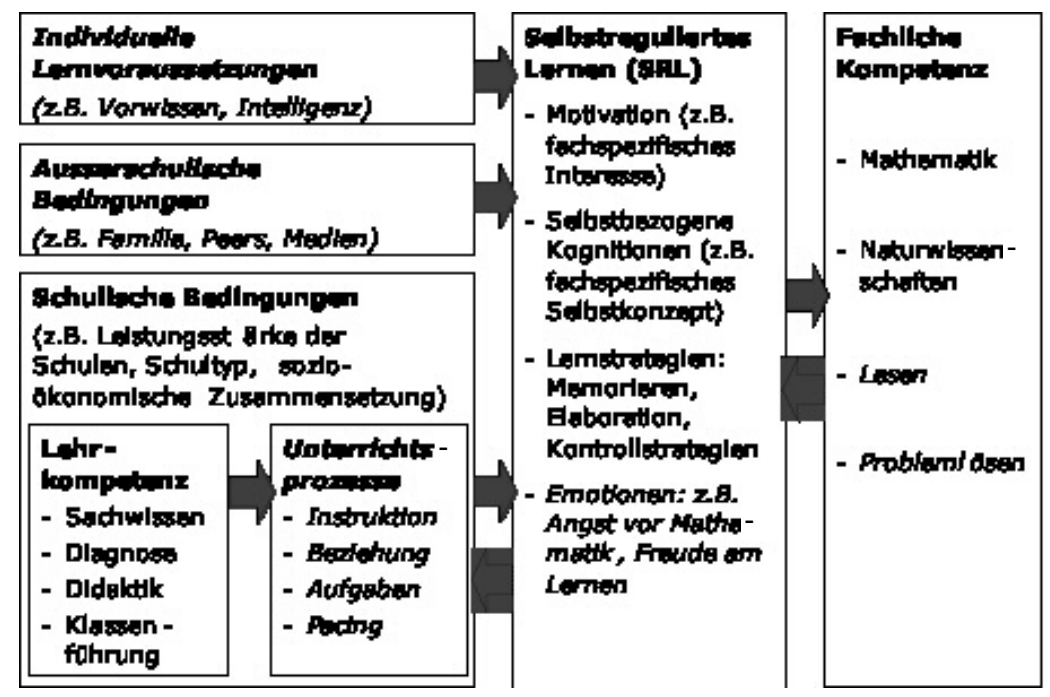

Abbildung 1: Selbstreguliertes Lernen (SRL) als Ziel und Bedingung schulischen Lernens. (Die kursiv gesetzten Bereiche bzw. Merkmale wurden in den beiden Studien für den vorliegenden Artikel nicht untersucht.)

Der Erwerb fachlicher und selbstregulativer Kompetenzen hängt von zahlreichen individuellen, schulischen und ausserschulischen Bedingungsfaktoren ab. Dabei nimmt die Lehrperson eine entscheidende Rolle für das Gelingen von Lernprozessen ein (Helmke \& Weinert, 1997). Das vorliegende Modell fokussiert auf die Kompetenzen der Lehrpersonen als Teil der schulischen Bedingungen. Lehrkompetenzen werden im Sinne handlungsleitender Kognitionen als eine wesentliche Voraussetzung für die Realisierung erfolgreicher Unterrichtsprozesse betrachtet. Damit wird zugleich offensichtlich, dass die Lehrkompetenzen den Lernertrag der Schülerinnen und Schüler nicht direkt beeinflussen, sondern über unterrichtliche Lehr-Lernprozesse und individuelle Lernaktivitäten vermittelt werden. In der Terminologie des Angebots-Nutzungs-Modells bieten Lehrpersonen im Unterricht Lerngelegenheiten an, welche die Schülerinnen und Schüler nutzen können. «Ob und wie effizient dieses Angebot genutzt wird, hängt von einer Vielzahl dazwischen liegender Faktoren ab» (Helmke, 2003, S. 41). $\mathrm{Zu}$ den schulischen Bedingungsfaktoren zählen auch Kontextfaktoren wie die Leistungsstärke oder die Heterogenität der Schülerschaft, die sich auf individuelle Lehr-Lernprozesse an Schulen auswirken können. 
Nachfolgend werden mit dem SRL und den Lehrkompetenzen jene beiden Bereiche weiter ausgeführt, die in diesem Beitrag im Zentrum stehen.

\section{Selbstreguliertes Lernen}

Der Begriff des SRL ist keineswegs einheitlich definiert, sondern steht vielmehr als Sammelbezeichnung für eine ganze Reihe von Teilprozessen des Lernens. Gemeinsam ist den verschiedenen Ansätzen jedoch eine konstruktivistische Sichtweise, indem sie selbstreguliert Lernende «als aktive Gestalter ihres eigenen Lernprozesses ansehen, die ihren Lernprozess mittels einer selbstbezogenen Feedbackschleife steuern» (Leopold \& Leutner, 2004, S. 365). Nach Artelt et al. (2003) zeichnen sich selbstreguliert Lernende dadurch aus, dass sie zum Lernen motiviert sind, passende Lernziele auswählen, das eigene Lernen steuern und überwachen sowie für die zu bewältigende Aufgabe geeignete Lernstrategien einsetzen. Es handelt sich folglich um eine komplexe Handlungskompetenz an der kognitive, metakognitive und motivational-emotionale Ressourcen beteiligt sind (Artelt, Demmerich \& Baumert, 2001).

Die Bedeutung der verschiedenen Aspekte des SRL für den Erwerb fachlicher Kompetenzen konnte wiederholt nachgewiesen werden (Artelt et al., 2003). Köller und Schiefele (2003) betonen jedoch auch, dass es relativ wenige gesicherte Befunde gibt, die eigenständige Effekte des SRL auf Wissenserwerbsprozesse auch gegen konkurrierende Prädiktoren nachweisen. Insbesondere zwischen selbst berichtetem Einsatz von Lernstrategien und Lernerfolg bestehen mehrheitlich niedrige und unklare Zusammenhänge (Leopold \& Leutner, 2004). Dieser an sich erwartungswidrige Befund kann dadurch erklärt werden, dass oft wenig valide Fragebogenerhebungen durchgeführt werden (z.B. Artelt, 2000). Höhere und konsistentere Korrelationen zwischen dem Einsatz von Lernstrategien und Lernerfolg zeigen sich, wenn Lernstrategien in konkreten Lernsituationen erfasst werden (Leopold \& Leutner, 2002). Leopold und Leutner (2004) betonen zudem, dass die Qualität des Lernstrategieeinsatzes für das Lernergebnis entscheidend sei.

Damit angemessene kognitive und metakognitive Lernstrategien überhaupt eingesetzt werden, bedarf es einer hinreichenden Motivation. Vor allem bereichsspezifische Interessen beeinflussen die kognitiven und emotionalen Prozesse während des Lernens positiv (Schiefele \& Schreyer, 1994). Dabei wird angenommen, dass motivationale Faktoren einen indirekten Einfluss über eine vertiefte kognitive Verarbeitung auf den Lernerfolg ausüben (Leopold \& Leutner, 2004).

Bedeutsame Effekte auf den Lernerfolg lassen sich auch für bereichsspezifische Fähigkeitsselbstkonzepte nachweisen (Marsh, 1987). Köller und Baumert (2001) konnten zeigen, dass das mathematische Selbstkonzept der Begabung in der siebenten Jahrgangsstufe auch bei Konstanthaltung wichtiger konkurrierender Prädiktoren (z.B. Vorwissen und Schultyp) einen signifikanten Einfluss auf 
die drei Jahre später gemessene Mathematikleistung aufweist. Dieses Ergebnis weist darauf hin, dass zwischen akademischen Selbstkonzepten und Leistungen eine wechselseitige Beziehung besteht, wonach die Leistung nicht nur das Selbstkonzept beeinflusst, sondern umgekehrt auch das Selbstkonzept die Leistung. Nach Jagacinski und Nicholls (1990) wirkt sich das Selbstkonzept auch auf die Motivation aus, da diese davon abhänge, ob jemand an seine eigene Kompetenz glaubt und sich auch bei auftretenden Schwierigkeiten weiter anstrengt (Schunk, 1990).

Die Ergebnisse von PISA 2003 bestätigen die Bedeutung der verschiedenen Aspekte des SRL für den Erwerb mathematischer Kompetenzen auch für die Schweiz (Brühwiler \& Biedermann, 2005). Höhere mathematische Kompetenzen hängen insbesondere mit einem positiven Selbstkonzept, geringerer Ängstlichkeit vor Mathematik und höherem Interesse zusammen. Ein solches günstiges Lernmuster zeigt sich stärker bei Knaben und erklärt einen erheblichen Teil der geschlechterspezifischen Leistungsdifferenzen in Mathematik. Ausserdem ergab sich, dass die Ausprägung verschiedener Aspekte des SRL auch vom Anspruchsniveau des besuchten Schultyps abhängt.

Schulorganisationsformen mit äusserer Differenzierung nach Leistungsgruppen haben zum Ziel, die Schülerinnen und Schüler in relativ homogenen Leistungsgruppen zu unterrichten. Solche gegliederten Schulsysteme sind vor allem in der Deutschschweiz verbreitet und beeinflussen das schulische Lernen in differenter Art und Weise. Sie führen einerseits dazu, dass in gymnasialen Schultypen höhere Lernzuwächse erreicht werden als in Schulen mit niedrigeren Ansprüchen (Baumert, Köller \& Schnabel, 2000; Gruehn, 2000). Andererseits weisen zahlreiche Untersuchungen darauf hin, dass separative Schulsysteme vor allem beim Übertritt in gymnasiale Ausbildungsgänge zu sozialen Benachteiligungen führen (z.B. Moser \& Berweger, 2005; Ramseier \& Brühwiler, 2003). Köller und Baumert (2001) berichten zudem über psychosoziale Kosten für Schülerinnen und Schüler der leistungsstärkeren Schulen. Aufgrund ungünstig interpretierter sozialer Vergleichsprozesse mit der eigenen leistungsstarken Bezugsgruppe verfügen Schülerinnen und Schüler mit gleichen individuellen Leistungen in leistungsstärkeren Schulen über geringere akademische Selbstkonzepte als jene in leistungsschwächeren Schulen. Dieses als big-fish-little-pond-effect (BFLPE; Marsh, 1987, 1990) oder Fischteich-Effekt bekannte Phänomen konnte auch für bereichsspezifische Interessen nachgewiesen werden (Köller et al., 2000). Der BFLPE auf Interesse wird dabei hauptsächlich über das Selbstkonzept vermittelt.

Die Schweizer PISA-Ergebnisse deuten ebenfalls auf einen BFLPE hin, denn das mathematische Selbstkonzept unterscheidet sich trotz erheblicher Leistungsdifferenzen kaum zwischen den verschiedenen Schultypen (Brühwiler \& Biedermann, 2005). Systematisch untersucht, d.h. unter Berücksichtigung des mittleren Leistungsniveaus der Schule und unter Konstanthaltung der individuellen Leistung, wurde der BFLPE jedoch noch nicht. Offen ist überdies, welche Be- 
deutung der Leistungsstärke von Schulen für den Einsatz von Lernstrategien zukommt.

\section{Adaptive Lehrkompetenz}

Der Begriff Adaptivität wird in der bestehenden Forschungsliteratur vor allem in zwei Kontexten verwendet: zum einen im Zusammenhang mit E-Learning und zum anderen im Konzept des adaptiven Lehrens. Nach Shute \& Towle (2003) kommt das Potential des computergestützten Lernens dann zum Vorschein, wenn eine optimale Passung zwischen Lernangebot und Lernunterstützung sowie den Bedürfnissen, Voraussetzungen und Möglichkeiten seitens der Lernenden hergestellt werden kann. In der Auffassung von Wang wird Adaptivität allgemeiner auf das Lehren bezogen. Sie versteht unter adaptivem Lehren «the use of alternative instructional strategies and resources to meet the learning needs of individual students» (Wang, 1980, S. 122).

Beide Forschungsstränge können als logische Fortsetzung der Aptitude-Treatment-Interaction-Forschung (ATI) der sechziger und siebziger Jahre des 20. Jahrhunderts (Cronbach \& Snow, 1977) gesehen werden. Ihnen ist gemeinsam, dass im Lernprozess den verschiedenen Lernvoraussetzungen besser Rechnung getragen werden soll als es im traditionellen Unterricht der Fall ist.

Das Konzept der adaptiven Lehrkompetenz (Beck et al., 2006) wie er in dieser Untersuchung verwendet wird, knüpft an die oben beschriebenen Adaptivitätsbegriffe an. Adaptive Lehrkompetenz bezeichnet demnach die Fähigkeit einer Lehrperson, die Planung und Durchführung des Unterrichts so auf die individuellen Lernvoraussetzungen der Schülerinnen und Schüler auszurichten und während des Unterrichts laufend anzupassen, dass für möglichst viele Schülerinnen und Schüler bestmögliche Bedingungen für das Erreichen der Lernziele geschaffen werden. Anders als bei der Erforschung von Unterrichtsprozessen stehen die handlungsleitenden Kognitionen der Lehrpersonen als Bedingung für gelingenden Unterricht im Zentrum des Interesses. Es wird untersucht, inwiefern sich Professionswissen im Unterrichtshandeln umsetzen lässt und auf den Lernerfolg der Schülerinnen und Schüler auswirkt.

Als Voraussetzung für eine hohe adaptive Lehrkompetenz sind in Anlehnung an Weinert (1996) vier verschiedene Kompetenzbereiche (Dimensionen) von Bedeutung:

1. ein umfassendes, klar strukturiertes und flexibel nutzbares Sachwissen (Sachkompetenz);

2. die Fähigkeit, die Schülerinnen und Schüler bezogen auf ihre Lernvoraussetzungen und ihre Lernergebnisse genau einzuschätzen (diagnostische Kompetenz);

3. ein reichhaltiges methodisch-didaktisches Wissen und Können (didaktische Kompetenz);

4. die Fähigkeit, eine Klasse so zu führen und zu begleiten, dass das Lernen 
nicht durch Störungen und Konflikte beeinträchtigt wird und die Unterrichtszeit bestmöglich genutzt werden kann (Klassenführungskompetenz) .

Das Konzept der adaptiven Lehrkompetenz umfasst eine Planungs- und eine Handlungsadaptivität. Mit adaptiver Planungskompetenz ist die Fähigkeit der Lehrperson gemeint, bei der Planung von Unterricht verschiedene Voraussetzungen (z.B. Sachstruktur, Fachdidaktik, individuelle Lernvoraussetzungen der Schülerinnen und Schüler) für einen gelingenden Lernprozess angemessen zu berücksichtigen. Die adaptive Handlungskompetenz bezieht sich darauf, wie Lehrpersonen unterschiedliche Informationen und Situationsveränderungen im Unterricht erfassen und in ihre Handlungsentscheidungen einbeziehen, um eine bestmögliche Unterstützung der individuellen Lernprozesse zu gewährleisten. Beides zusammen kennzeichnet die Anpassungsfähigkeit des Lehrerhandelns in Bezug auf die vielfältigen Bedingungen von Lehr-Lern-Situationen. Die Berück sichtigung der verschiedenen Perspektiven und Wissensbereiche zur Planung und Durchführung von Unterricht erfordert von den Lehrpersonen hohe metakognitive Kompetenzen.

\section{Fragestellung}

Die Schweizer PISA-Analysen haben gezeigt, dass Schülerinnen und Schüler mit hohen mathematischen Kompetenzen über günstigere affektive und motivationale Lernvoraussetzungen verfügen (Brühwiler \& Biedermann, 2005). In Bezug auf die Anwendung von Lernstrategien ist das Bild uneinheitlich. Einzig der häufige Einsatz von Memorierstrategien korreliert in allen Kantonen negativ mit den Mathematikleistungen. Es ergaben sich zudem Hinweise darauf, dass die Ausprägung verschiedener Aspekte des SRL und deren Beziehungen zur fachlichen Leistung auch vom besuchten Schultyp abhängen. Ergänzend stellt sich die Frage, ob auch die Leistungsstärke der Schulen innerhalb der Schultypen für die Merkmale des SRL eine Rolle spielen.

Die erste Fragestellung bezieht sich somit auf die Bedeutung des Schultyps und des Leistungsniveaus der Schulen für verschiedene Aspekte des SRL. Dabei interessiert, ob sich anhand der PISA-Daten auch ein BFLPE für die Schweiz nachweisen lässt. Es ist zu vermuten, dass aufgrund ungünstiger sozialer Vergleichsprozesse die mittlere Schulleistung einen negativen Effekt auf das mathematische Selbstkonzept aufweist, wenn die individuelle Mathematikleistung konstant gehalten wird. Zusätzlich zum mathematischen Selbstkonzept und zum Interesse werden auch die Lernstrategien als abhängige Variablen von der individuellen Mathematikleistung und der mittleren Schulleistung untersucht. Mit dieser ersten Studie soll ein Beitrag zur Aufhellung der teilweise unerwarteten bzw. uneinheitlichen Zusammenhänge zwischen der Lernstrategieanwendung und den Leistungen geleistet werden. 
Vor dem Hintergrund der mehrheitlich positiven Effekte des SRL soll in einer zweiten Studie geklärt werden, welche Kompetenzen von Lehrpersonen sich für die Entwicklung des SRL als günstig erweisen. Dabei wird erwartet, dass eine hohe Ausprägung der adaptiven Lehrkompetenz die Entwicklung der SRLMerkmale positiv beeinflusst. Eine angemessene Berücksichtigung individueller kognitiver und motivationaler Lernvoraussetzungen während dem Unterricht dürfte sich vorteilhaft auf relevante Lerneinstellungen der Schülerinnen und Schüler auswirken. Da bei PISA keine Daten von Lehrpersonen vorliegen, wird diese Fragestellung anhand von Daten aus dem Nationalfonds-Projekt «Adaptive Lehrkompetenz» (Beck et al., 2006) analysiert.

\section{PISA 2003: Bedeutung des Schultyps und der Lej- stungsstärke von Schulen für das SRL (Studie 1)}

\section{Methodisches Vorgehen \\ Überblick}

Die Datengrundlage beruht auf der Schweizer Stichprobe der neunten Klassenstufen von PISA 2003. Ausführliche Beschreibungen zum methodischen Vorgehen bei PISA finden sich in den nationalen und internationalen Berichten (OECD, 2004; Zahner Rossier, 2005). Da die Bedeutung des Schultyps für das SRL eine zentrale Fragestellung dieser Studie darstellt, wurden für die Analysen nur jene Schülerinnen und Schüler berücksichtigt, die eindeutig einem der drei Schultypen Grundansprüche (z.B. Realschule), erweiterte Ansprüche (z.B. Sekundarschule) oder hohe Ansprüche (z.B. Gymnasium) zugewiesen werden konnten. Ausgeschlossen wurden kleine Schulen mit weniger als 10 Schülerinnen und Schülern mit gültigen Werten in den untersuchten Variablen. Dies bedeutet, dass die Ergebnisse nicht ohne Weiteres auf kleine Schulen, die oft besondere Merkmale wie z.B. Mehrklassenunterricht aufweisen, generalisierbar sind. Von den 21'634 bei PISA getesteten Schülerinnen und Schülern verbleiben somit 15'575 Schülerinnen und Schüler aus 272 Schulen in der Stichprobe. Aufgrund dieser grossen Stichprobe werden schon verhältnismässig kleine Regressionsgewichte statistisch signifikant. Für eine angemessene Interpretation der Effekte ist deshalb die Grösse der Regressionskoeffizienten aussagekräftiger als die statistischen Signifikanzwerte.

\section{Instrumente}

Die bei PISA erfassten Aspekte des SRL stützen sich auf das Rahmenmodell von Boekaerts (1997, 1999) und lassen sich den Bereichen selbstbezogene Fähigkeitskognitionen, motivationale Orientierung und Lernstrategien zuordnen. Die Erfassung erfolgte über einen Fragebogen mit vierstufigen Antwortformaten $(1=$ stimme überhaupt nicht zu; 4 = stimme völlig zu), in welchem die Schülerinnen und Schüler ihr Lernverhalten einschätzten. 
Für die vorliegenden Analysen wurden fünf Skalen ${ }^{2}$ ausgewählt. Aus dem Bereich selbstbezogene Fähigkeitskognitionen wurde das mathematisches Selbstkon$z e p t$ ausgewählt. Damit wird erfasst, wie stark die Schülerinnen und Schüler von ihren mathematischen Fähigkeiten überzeugt sind. Das Interesse an Mathematik gehört zum Bereich der intrinsischen motivationalen Orientierung. Den Lernstrategien lassen sich drei Skalen zuordnen, nämlich Memorierstrategien (Formen des Auswendiglernens), Elaboration (Verbinden von neuen Inhalten mit bestehenden Wissensstrukturen) und Kontrollstrategien (metakognitive Strategien zur Planung, Überwachung und Regulierung des eigenen Lernens).

Aufgrund dieser selbstberichteten Lernermerkmale lässt sich zwar nicht unmittelbar darauf schliessen, ob und in welcher Qualität die Schülerinnen und Schüler ihr Lernen in spezifischen Situationen selbst regulieren. Nach Artelt et al. (2003) kann jedoch angenommen werden, dass dies bei Schülerinnen und Schülern mit hohen Ausprägungen in diesen Merkmalen häufiger vorkommt.

Die individuelle und auf Schulebene aggregierte Mathematikleistung beruht auf der Gesamtskala Mathematik von PISA 2003. Diese umfasst die mathematischen Gebiete Raum und Form (vergleichbar mit Geometrie), Veränderungen und Beziehungen (Arithmetik), quantitatives Denken (Algebra) und Unsicherheit (Statistik und Wahrscheinlichkeitsrechnung). Es wurde jeweils nur der erste der fünf plausiblen Werte (Mislevy, Johnson \& Muraki, 1992; OECD, 2005) in die Analyse einbezogen.

\section{Statistisches Vorgehen}

Die vorgesehene Hypothesenprüfung erfordert eine gemeinsame Modellierung von Individual- und Schulmerkmalen, weshalb für die Analysen Zweiebenenmodelle spezifiziert werden. Auf Individualebene werden die Merkmale des SRL, die Mathematikleistung sowie die Schultypen eingeführt. Letztere werden deswegen zu den individuellen Merkmalen gezählt, weil an den Schweizer Schulen oft verschiedene Schultypen angeboten werden. Als Prädiktor der Schulebene wird die durchschnittliche Mathematikleistung einbezogen. Damit kann der Einfluss der Leistungsstärke der Schulen von den individuellen Effekten getrennt werden. Das mehrebenenanalytische Vorgehen hat zudem den Vorteil einer adäquaten Berücksichtigung des zweistufigen Stichprobenverfahrens, bei dem zuerst Schulen und erst dann einzelne Schülerinnen und Schüler gezogen werden (Lüdtke, Köller, Bundt, Gomolka \& Waterman, 2004).

Die Mehrebenenanalysen wurden mit dem Programm HLM 6 (Raudenbush, Bryk, Cheong, Congdon \& du Toit, 2004) durchgeführt. Die Analysen erfolgten mit ungewichteten Daten, da nicht Aussagen über ganze Populationen angestrebt werden, sondern Zusammenhänge zwischen schulischen Leistungen und Lernermerkmalen im Vordergrund stehen. Sowohl die Prädiktorvariablen mit Ausnahme des dummycodierten Schultyps - als auch die Kriteriumsvariablen wurden z-standardisiert. Die Regressionskoeffizienten können somit wie standardisierte Koeffizienten einer einfachen Regressionsanalyse interpretiert 
werden. Die Schultypen wurden wie folgt kodiert: GYM (hohe Ansprüche = 1; andere $=0$ ) und REAL (Grundansprüche $=1$; andere $=0$ ). Referenzkategorie ist demzufolge der Schultyp erweiterte Ansprüche.

\section{Ergebnisse}

In Tabelle 1 sind die Ergebnisse der verschiedenen Mehrebenenmodelle für die abhängigen Variablen mathematisches Selbstkonzept und Interesse dargestellt. Die Modelle M1 bis M3 bestätigen den BFLPE für das mathematische Selbstkonzept eindrücklich. Unter Konstanthaltung der individuellen Leistung fällt bei M1 der Effekt der durchschnittlichen Schulleistung auf das Selbstkonzept deutlich negativ aus $(\beta=-.46)$. Schülerinnen und Schüler aus leistungsstarken Schulen verfügen bei gleichen individuellen Mathematikleistungen über ein geringeres Selbstkonzept als Schülerinnen und Schüler aus leistungsschwächeren Schulen. Die individuelle Leistung hat hingegen nach Kontrolle der Leistungsstärke der Schulen einen positiven Effekt auf das Selbstkonzept $(\beta=.46)$. Dieser Effekt fällt gegenüber einer einfachen Regressionsanalyse $(\beta=.32)$ stärker aus. Dies macht nochmals den Mehrwert mehrebenenanalytischer Auswertungsverfahren und der Kontrolle von Kontextmerkmalen deutlich. Wird das mittlere Leistungsniveau der Schule nicht konstant gehalten, führt dies zu einer Unterschätzung des Zusammenhangs zwischen individueller Leistung und Selbstkonzept.

Wenn anstelle der mittleren Schulleistung die Schultypen kontrolliert werden (M2), fällt der Effekt der individuellen Mathematikleistung auf das Selbstkonzept mit $\beta=-.57$ sogar noch etwas stärker aus. Insgesamt erklären die ins Modell aufgenommenen Prädiktoren 18 Prozent der Varianz. Die unstandardisierten Regressionskoeffizienten der dummycodierten Schultypen können jeweils als Mittelwertsabweichungen zum Schultyp erweiterte Ansprüche interpretiert werden und belegen wiederum den BFLPE. Schülerinnen und Schüler in gymnasialen Schultypen weisen bei Konstanthaltung der individuellen Mathematikkompetenz ein signifikant niedrigeres Selbstkonzept auf. Durch die gleichzeitige Berücksichtigung des Schultyps und der durchschnittlichen Schulleistung (M3) verändert sich gegenüber M1 und M2 einzig der Regressionskoeffizient der Schulebene markant (von $\beta=-.46$ auf $\beta=-.22$ ). Die Leistungsstärke der Schulen hat nach Kontrolle der Schultypen einen geringeren negativen Effekt auf das Selbstkonzept, ist aber auch innerhalb der Schultypen bedeutsam. Der Effekt der Schultypzugehörigkeit ist nach wie vor beträchtlich. Im gegenüber M2 marginalen Anstieg der insgesamt erklärten Varianz auf 19 Prozent wird ersichtlich, dass die mittlere Schulleistung zu einem grossen Teil durch den Schultyp erklärt wird.

Zusätzlich zu den bisher dargestellten Modellen wurde ein möglicher Effekt der sozialen Herkunft auf das mathematische Selbstkonzept geprüft, da in den bisherigen PISA-Befunden durchwegs ein hoher Zusammenhang der sozialen Herkunft mit der fachlichen Leistung gefunden wurde. Ein (hier nicht dargestelltes) erweitertes Modell ergab weder einen signifikanten Effekt der sozialen 
Herkunft auf das mathematische Selbstkonzept noch nennenswerte Veränderungen der übrigen Koeffizienten. Daraus lässt sich folgern, dass die soziale Herkunft keinen eigenständigen Erklärungswert für das bereichsspezifische Selbstkonzept aufweist.

Tabelle 1: Mehrebenenanalytische Ergebnisse zur Vorhersage des mathematischen Selbstkonzepts und Interesses.

\begin{tabular}{|c|c|c|c|c|c|c|c|c|c|c|}
\hline \multirow{3}{*}{$\begin{array}{l}\text { Abhängige Variable } \\
\text { Modell }\end{array}$} & \multicolumn{6}{|c|}{ Selbstkonzept in Mathematik } & \multicolumn{4}{|c|}{ Interesse an Mathematik } \\
\hline & \multicolumn{2}{|c|}{ M1 } & \multicolumn{2}{|c|}{ M2 } & \multicolumn{2}{|c|}{ M3 } & \multicolumn{2}{|c|}{ M4 } & \multicolumn{2}{|c|}{ M5 } \\
\hline & $\beta$ & $\mathrm{p}$ & $\beta$ & $\mathrm{p}$ & $\beta$ & $\mathrm{p}$ & $\beta$ & $\mathrm{p}$ & $\beta$ & $\mathrm{p}$ \\
\hline \multicolumn{11}{|l|}{ Individualebene } \\
\hline $\begin{array}{l}\text { Individuelle Mathematik- } \\
\text { leistung }\end{array}$ & .46 & $<.01$ & .57 & $<.01$ & .58 & $<.01$ & .27 & $<.01$ & .38 & $<.01$ \\
\hline Hohe Ansprüche (GYM) & & & -.44 & $<.01$ & -.38 & $<.01$ & & & -.39 & $<.01$ \\
\hline Grundansprüche (REAL) & & & .50 & $<.01$ & .46 & $<.01$ & & & .40 & $<.01$ \\
\hline \multicolumn{11}{|l|}{ Schulebene } \\
\hline $\begin{array}{l}\text { Durchschnittliche Mathe- } \\
\text { matikleistung der Schule }\end{array}$ & -.46 & $<.01$ & & & -.22 & $<.01$ & -.32 & $<.01$ & -.10 & $<.01$ \\
\hline $\mathrm{R}^{2}$ & \multicolumn{2}{|c|}{.15} & \multicolumn{2}{|c|}{.18} & \multicolumn{2}{|c|}{.19} & \multicolumn{2}{|c|}{.05} & \multicolumn{2}{|c|}{.09} \\
\hline
\end{tabular}

Anmerkung: $\mathrm{N}=15^{\prime} 575$. Die Regressionskoeffizienten $(\beta)$ sind für kontinuierliche Prädiktoren standardisiert, für die dummycodierten Variablen des Schultyps unstandardisiert (Referenzkategorie «erweiterte Ansprüche»).

Das Modell M4 weist in Übereinstimmung mit Köller et al. (2000) auch für das Interesse an Mathematik einen BFLPE nach. Der Effekt ist verglichen mit dem Selbstkonzept etwas schwächer. Während der Regressionskoeffizient der aggregierten Leistung mit $\beta=-.32$ negativ ausfällt, ist er für die individuelle Leistung positiv $(\beta=.27)$. Der starke Rückgang des Regressionskoeffizienten der mittleren Schulleistung (von $\beta=-.32$ auf -.10) bei M5 deutet darauf hin, dass der BFLPE vor allem über die Schultypzugehörigkeit vermittelt ist. Innerhalb der Schultypen spielt das Leistungsniveau der Schule eine untergeordnete Rolle. Wie zuvor beim mathematischen Selbstkonzept zeigt sich auch hier, dass die individuelle Leistung enger mit dem Interesse zusammenhängt, wenn die Kontextfaktoren berücksichtigt werden. Wird zusätzlich das mathematische Selbstkonzept mit modelliert, so bestätigt sich der Befund von Köller et al. (2000), dass der BFLPE auf das Interesse hauptsächlich über das bereichsspezifische Selbstkonzept vermittelt wird. ${ }^{3}$

Die Modelle M6 bis M11 untersuchen die Bedeutung der mittleren Schulleistung und der Schultypzugehörigkeit auf die Beziehung zwischen individueller Leistung und dem selbstberichteten Einsatz von Lernstrategien (vgl. Tabelle 2). 
Insgesamt sind die Effekte mit einer maximal erklärten Varianz von 3 Prozent deutlich schwächer als beim mathematischen Selbstkonzept und Interesse. In M6 wird die mittlere Schulleistung konstant gehalten. Der negative Regressionskoeffizient für die individuelle Mathematikleistung $(\beta=-.15)$ verweist auf einen negativen Zusammenhang von Memorierstrategien mit fachlichen Leistungen. Anders formuliert besagt dies, dass Memorierstrategien häufiger von Schülerinnen und Schülern mit schwächeren Leistungen gewählt werden, was den Befund aus den nationalen PISA-Analysen (Brühwiler \& Biedermann, 2005) bestätigt. Der Einbezug der Schultypen in M7 führt zu keiner Veränderung dieses Effekts. Strategien des Auswendiglernens werden in allen Schultypen gleich häufig eingesetzt und hängen nicht vom Leistungsniveau der Schule ab.

M8 zeigt, dass Elaborationsstrategien häufiger an Schulen mit niedrigeren Mathematikleistungen angewendet werden $(\beta=-.22)$. Dieser Effekt verschwindet unter Berücksichtigung der Schultypen weitgehend (M9) und lässt sich dadurch erklären, dass in gymnasialen Ausbildungsgängen seltener Elaborationsstrategien angewendet werden als von Schülerinnen und Schülern mit Grundansprüchen. Die Berücksichtigung des Schultyps und des mittleren Leistungsniveaus der Schulen führt dazu, dass - wie theoretisch erwartet - ein vermehrter Einsatz von Elaborationsstrategien mit etwas höheren individuellen Leistungen einhergeht.

Tabelle 2: Mehrebenenanalytische Ergebnisse zur Vorhersage des Einsatzes von Lernstrategien

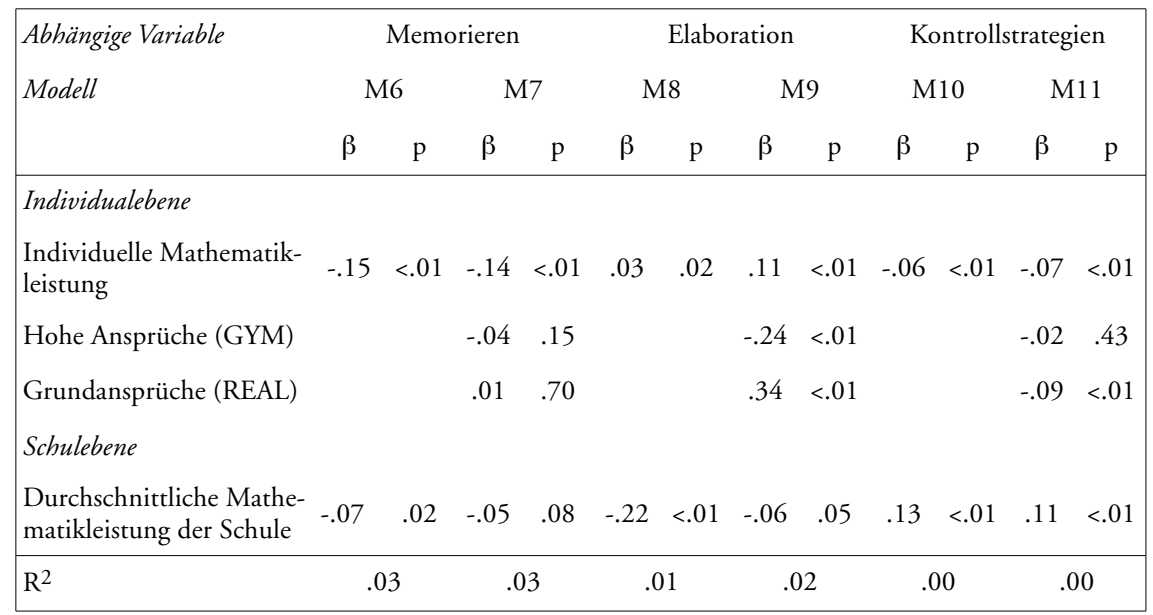

Anmerkung: $N=15$ '575. Die Regressionskoeffizienten $(\beta)$ sind für kontinuierliche Prädiktoren standardisiert, für die dummycodierten Variablen des Schultyps unstandardisiert (Referenzkategorie «erweiterte Ansprüche»). 
Dem geringfügig negativen Zusammenhang der individuellen Leistung mit dem Einsatz von Kontrollstrategien (M10 und M11) steht ein leicht positiver Effekt bei der mittleren Schulleistung gegenüber. An leistungsstärkeren Schulen werden demnach etwas häufiger Kontrollstrategien eingesetzt. Dieser Effekt bleibt mit $\beta=.11$ bestehen, wenn der Schultyp eingeführt wird (M11). Der schwach negative Effekt des Schultyps Grundansprüche $(\beta=-.09)$ und der nicht signifikante Effekt der gymnasialen Ausbildungstypen $(\beta=-.02)$ deuten auf einen umgekehrt u-förmigen Zusammenhang hin. Schülerinnen und Schüler aus Schultypen mit erweiterten Ansprüchen geben etwas häufiger an, Kontrollstrategien einzusetzen.

\section{Förderung des SRL durch adaptive Lehrkompetenz (Studie 2)}

Die vertieften Analysen der PISA-Daten (Studie 1) haben nochmals die Bedeutung der verschiedenen Merkmale des SRL für die individuelle Leistungsfähigkeit belegt. Dies gilt insbesondere für das mathematische Selbstkonzept und Interesse. Studie 2 geht nun der Frage nach, welche Kompetenzen der Lehrpersonen eine positive Entwicklung der SRL-Merkmale begünstigen könnten.

Die empirische Grundlage zur Überprüfung des Einflusses von Lehrkompetenzen auf die Förderung des SRL bilden Daten aus dem Projekt «Adaptive Lehrkompetenz» (Beck et al., 2006). Mit unterschiedlichen methodischen Zugängen wurde die adaptive Lehrkompetenz erfasst und deren Wirkung auf verschiedene Aspekte des SRL bei den Schülerinnen und Schülern untersucht. Obgleich das Projekt "Adaptive Lehrkompetenz» im Fachbereich Naturwissenschaften durchgeführt wurde, dürften die Befunde auf andere bereichsspezifische Lernermerkmale übertragbar sein.

\section{Methodisches Vorgehen \\ Stichprobe und Durchfïhrung}

Die vorliegende Teilstudie basiert auf Daten von insgesamt 49 Lehrpersonen und 890 Schülerinnen und Schülern. 26 Lehrpersonen unterrichteten in der Primarstufe (4. und 5. Klassenstufen), 23 in der Sekundarstufe I (7. und 8. Klassenstufen). Für die Teilnahme an der Studie wurde vorausgesetzt, dass die Lehrpersonen in der untersuchten Klasse mindestens teilweise im Fachbereich Naturwissenschaften unterrichten.

Um Veränderungen in der Entwicklung der SRL-Merkmale zu erfassen, wurden alle Erhebungen zu zwei Messzeitpunkten durchgeführt. Der Vortest erfolgte zu Beginn des Schuljahres (September 2003), der Nachtest gegen Ende des Schuljahres (Juni 2004). 


\section{Instrumente}

\section{Vignettentest zur Erfassung der adaptiven Planungskompetenz}

Für die Erhebung der adaptiven Planungskompetenz von Lehrpersonen wurde ein Vignettentest (Mason, 1994) entwickelt, der handlungsleitende Kognitionen bei der Unterrichtsplanung erfasst. Die Vignette enthält für die untersuchten Lehrpersonen die Handlungsaufforderung, sich in die Situation einer Praktikumslehrperson zu versetzen, die Studierenden bei der Unterrichtsvorbereitung helfen soll. Die Vorschläge zur Unterrichtsvorbereitung mussten begründet werden, um die Planungsüberlegungen transparent zu machen. Der Vignettentest erfolgte schriftlich und konnte von den Lehrpersonen ohne Zeitdruck gelöst werden.

Die Antworttexte wurden von zwei geschulten Personen nach einem detaillierten Auswertungsmanual im Einigungsverfahren codiert und im Hinblick auf drei Dimensionen der adaptiven Lehrkompetenz (Diagnose, Didaktik, Bedeutung der Sachkompetenz) quantifiziert. Die Dimension Klassenführung wurde weggelassen, weil Aspekte der Klassenführung bei der Planung einzelner Lektionen eine eher untergeordnete Rolle spielen und die Vignette nicht darauf ausgerichtet war.

\section{Videotest zur Erfassung der adaptiven Handlungskompetenz}

Die adaptive Handlungskompetenz von Lehrpersonen wurde mittels Videotest erhoben. Dieser wurde mit dem Ziel entwickelt, Grundlagen der adaptiven Handlungskompetenz möglichst unterrichtsnah und gebunden an konkrete Handlungskontexte, aber dennoch standardisiert zu erfassen. Im Videotest mussten die getesteten Lehrpersonen eine nach einem Drehbuch verfilmte Unterrichtssequenz zum Thema "Wasser- und Luftdruck» betrachten und unmittelbar angeben, an welcher Stelle sie anders als die Lehrperson im Video handeln würden. Vorgeschlagene Handlungsalternativen waren zu begründen. Der Videotest wurde als Einzeltest unter Anleitung geschulter Versuchleiterinnen durchgeführt. Die Antworten wurden analog zur Vignette im Einigungsverfahren codiert und im Hinblick auf drei Dimensionen der adaptiven Lehrkompetenz (Diagnose, Didaktik und Klassenführung) quantifiziert. Details zum Videotest finden sich bei Bischoff, Brühwiler und Baer (2005).

Obschon mit dem gewählten Vorgehen eine unmittelbare Entscheidungssituation simuliert wurde, ist die mit dem Videotest erfasste adaptive Handlungskompetenz nicht mit dem Handeln im eigenen Unterricht gleichzusetzen. Es wird aber angenommen, dass eine hohe Qualität der Unterrichtswahrnehmung und der vorgeschlagenen Handlungsoptionen zumindest bessere Voraussetzungen bietet, auch im eigenen Unterricht adaptiver zu agieren. Diese Annahme lässt sich mit Überlegungen von Gruber, Mandl und Renkl (2000) zum trägen bzw. anwendbaren Wissen stützen, wonach sich kontextgebundenes Wissen wie es im Videotest mindestens teilweise erfasst wurde - eher in Handeln überführen lässt als Wissen, das losgelöst von Kontexten repräsentiert wird. 
Fragebogen zur Erfassung der Merkmale des SRL

Analog zur Studie 1 wurden fünf SRL-Merkmale (fachspezifisches Selbstkonzept und Interesse, Memorieren, Elaborations- und Kontrollstrategien) ausgewählt. Die Itemformulierungen weichen teilweise geringfügig voneinander $a b$, da die Items aus dem Fragebogen von PISA 2000 (OECD, 2001) stammen und sprachlich auf den Fachbereich Naturwissenschaften angepasst wurden. Die Datenerhebungen bei den Schülerinnen und Schülern erfolgten nach standardisierter Anleitung durch die Lehrpersonen während der Unterrichtszeit. Die internen Konsistenzen der Skalen liegen zwischen Cronbachs $\alpha=.61$ und $\alpha=.86$.

\section{Statistisches Vorgehen}

Die vorliegenden Analysen haben zum Ziel, Effekte der Lehrpersonen auf die durchschnittliche Entwicklung des SRL bei ihren Klassen zu ermitteln. Die Auswertungen erfolgten mittels zweifaktoriellen Varianzanalysen mit Messwiederholung. Die Werte der Schülerinnen und Schüler wurden auf Klassenebene aggregiert. ${ }^{4}$ Aufgrund der vergleichsweise geringen Fallzahlen $(\mathrm{N}=49$ Lehrpersonen bzw. Klassen) sind jeweils auch die Effektgrössen (partielles $\eta^{2}$ ) für den Interaktionseffekt (Zeit x AL-Gruppe) angegeben. Gemäss Cohen (1988) sind Effektgrössen ab .01 als klein, ab .06 als mittel und ab .14 als gross zu bezeichnen.

\section{Bildung von Gruppen mit unterschiedlicher adaptiver Lehrkompetenz}

Als Mass für die Ausprägung der adaptiven Lehrkompetenz werden die Resultate des Vignettentests (adaptive Planungskompetenz) und des Videotests (adaptive Handlungskompetenz) verwendet. Auf der Basis der Nachtestergebnisse wurden die getesteten Lehrpersonen mittels hierarchischer Clusteranalyse zu zwei Gruppen mit ähnlichen Ausprägungen in den einzelnen Dimensionen der adaptiven Lehrkompetenz zugeteilt. Das in Abbildung 2 dargestellte Clusterprofil veranschaulicht die Ausprägungen in den einzelnen Dimensionen. Die Skalenmittelwerte entsprechen den durchschnittlich erreichten Prozentanteilen der Maximalpunktzahl pro Dimension. Ein Wert von 50 bedeutet demnach, dass genau die Hälfte der möglichen Punkte in einer bestimmten Dimension erreicht wurde.

Die Lehrpersonen der Gruppe «hohe adaptive Lehrkompetenz»(AL hoch) umfasst 17 Lehrpersonen und zeichnet sich in allen Dimensionen durch signifikant höhere Werte aus als die Gruppe «niedrige adaptive Lehrkompetenz» (AL niedrig; $\mathrm{N}=32$ ). Die geringste Differenz findet sich mit einem Mittelwertsunterschied von 14 Punkten bei der Klassenführung $(\mathrm{t}=2.89, \mathrm{df}=47, \mathrm{p}<.01)$. 


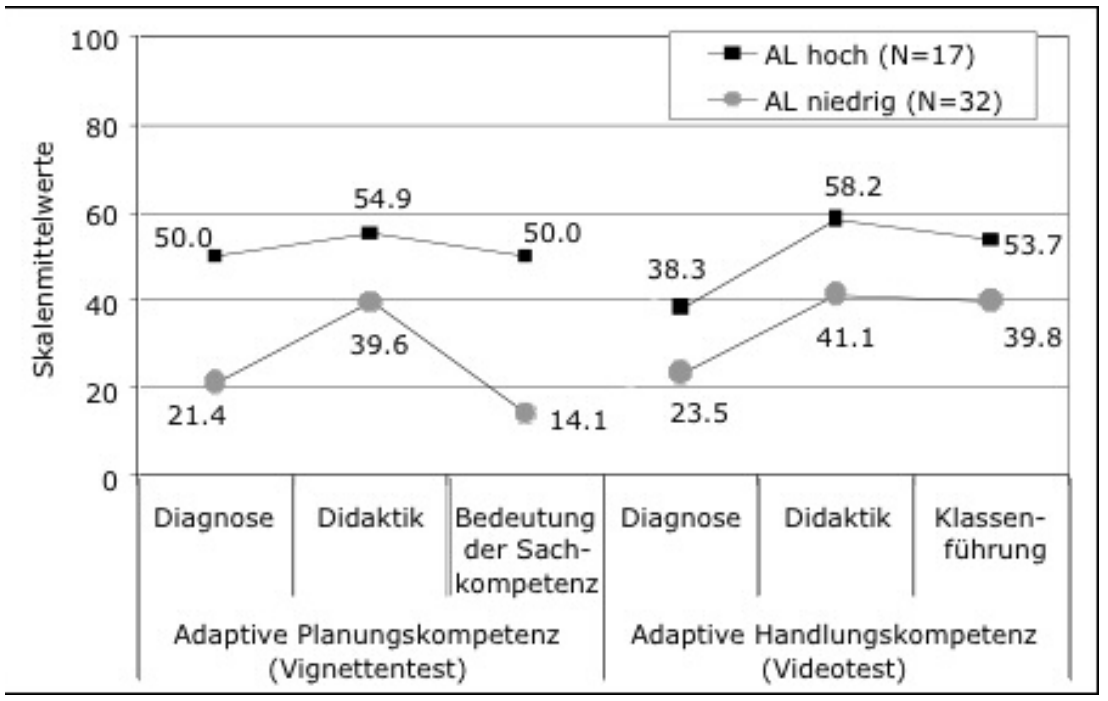

Abbildung 2: Skalenmittelwerte für die beiden Gruppen der adaptiven Lehrkompetenz (Clusterprofil).

Die mittels Clusteranalyse gebildeten Gruppen werden in den Analysen als dichotomes Merkmal zur Unterscheidung von Lehrpersonen mit hoher bzw. niedriger Ausprägung in der adaptiven Lehrkompetenz verwendet. Zur Unterscheidung von Lehrpersonengruppen bezüglich ihrer adaptiven Planungs- bzw. Handlungskompetenzen wurden zwei weitere Clusteranalysen durchgeführt, in welchen jeweils nur die Daten aus dem Vignetten- bzw. Videotest berücksichtigt wurden.

\section{Ergebnisse}

Aus Tabelle 3 geht hervor, dass die Selbsteinschätzung der verschiedenen Merkmale des SRL mit Ausnahme der Kontrollstrategien zwischen Vortest und Nachtest abnimmt. Diese Abnahme zeigt sich in der Oberstufe stärker als in der Primarstufe. Während in der Primarstufe nur die Memorierstrategien im Nachtest signifikant geringer ausfallen $(\mathrm{t}=2.24, \mathrm{df}=25, \mathrm{p}=.04)$, sinken in der Oberstufe das naturwissenschaftliche Interesse $(\mathrm{t}=4.71, \mathrm{df}=22, \mathrm{p}<.01)$ und Selbstkonzept $(\mathrm{t}=2.46, \mathrm{df}=22, \mathrm{p}=.02)$ sowie die Elaborationsstrategien $(\mathrm{t}=3.28, \mathrm{df}=$ $22, \mathrm{p}=.01)$. Die Einschätzungen des bereichsspezifischen Interesses und Selbstkonzepts sowie der Elaborationsstrategien sind generell in der Primarstufe signifikant positiver als in der Oberstufe. Bei den Memorier- und den Kontrollstrategien lässt sich kein Stufenunterschied nachweisen. Die Abnahme verschiedener Merkmale des SRL im Verlaufe der Schulzeit deckt sich mit Befunden von Artelt (2000) und speziell bezogen auf das Selbstkonzept mit Ergebnissen von Zimmerman und Martinez-Pons (1990). 
Tabelle 3: Ausprägung verschiedener Merkmale des SRL im Vor-und Nachtest

\begin{tabular}{|l|l|l|l|l|}
\hline & Messzeitpunkt & M & SD & p \\
\hline Fachspezifisches Selbstkonzept & Vortest & 2.85 & 0.25 & .02 \\
& Nachtest & 2.78 & 0.26 & \\
Fachspezifisches Interesse & Vortest & 2.93 & 0.28 & .03 \\
Memorieren & Nachtest & 2.86 & 0.33 & \\
Elaboration & Vortest & 2.90 & 0.22 & .02 \\
Kontrollstrategien & Nachtest & 2.83 & 0.24 & \\
& Vortest & 2.64 & 0.20 & $<.01$ \\
& Nachtest & 2.53 & 0.28 & \\
& Vortest & 3.24 & 0.16 & n.s. \\
\hline
\end{tabular}

Anmerkung: $\mathrm{N}=49$ Klassen. Signifikanzberechungen mittels t-Tests mit gepaarten Stichproben; n.s. = nicht signifikant.

Vergleicht man die Veränderung der SRL-Merkmale der Klassen von Lehrpersonen mit hoher bzw. niedriger adaptiver Lehrkompetenz, so weisen die Interaktionseffekte (Zeit x AL-Gruppe) zwar in die erwartete Richtung, erreichen jedoch die Signifikanzgrenze nicht. Der stärkste Effekt zu Gunsten der Lehrpersonen mit höheren adaptiven Lehrkompetenzen zeigt sich beim bereichsspezifischen Interesse $\left(\eta^{2}=.04\right)$, der schwächste Effekt beim Selbstkonzept $\left(\eta^{2}=.01\right)$.

Bemerkenswert sind die Ergebnisse, wenn nach adaptiver Handlungs- und Planungskompetenz unterschieden wird. Während sich die SRL-Merkmale in Abhängigkeit der beiden Lehrergruppen mit hoher bzw. niedriger adaptiver Planungskompetenz über das Schuljahr hinweg nicht signifikant verändern, zeigen sich bei der adaptiven Handlungskompetenz signifikante Effekte (Abbildung 3). In Klassen von Lehrpersonen mit hoher adaptiver Handlungskompetenz entwickeln sich bereichsspezifische Interessen $\left(\mathrm{F}=6.32, \mathrm{df}=1,45, \mathrm{p}=.02 ; \eta^{2}=\right.$ $.12)$ und Selbstkonzepte $\left(\mathrm{F}=3.12, \mathrm{df}=1,45, \mathrm{p}=.08\right.$; $\left.\eta^{2}=.06\right)$ signifikant ${ }^{5}$ besser als in Klassen von Lehrpersonen mit niedrigeren Werten in der adaptiven Handlungskompetenz. Lehrpersonen mit ausgeprägten adaptiven Handlungskompetenzen gelingt es besser, Schülerinnen und Schüler für den Unterricht zu motivieren und deren Selbstkonzept positiv zu beeinflussen. Der Einsatz von Lernstrategien konnte insgesamt nicht signifikant beeinflusst werden. Ein nennenswerter Effekt liegt bei den Elaborationsstrategien vor $\left(\eta^{2}=.02\right)$. Zudem zeigt sich auf der Primarstufe ein positiver Effekt adaptiver Handlungskompetenzen auf die häufigere Verwendung von Kontrollstrategien (Beck et al., 2006). 


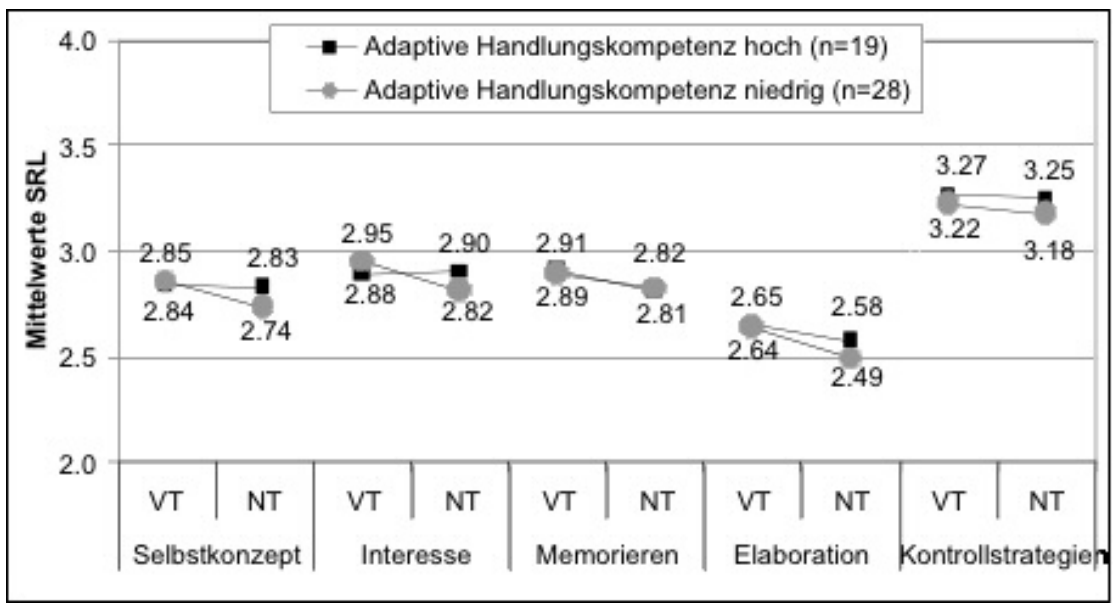

Abbildung 3: Veränderung verschiedener Merkmale des SRL zwischen Vortest (VT) und Nachtest (NT) nach adaptiver Handlungskompetenz der Lehrpersonen.

Für die Schulpraxis von besonderem Interesse ist die Frage, welche Dimensionen der adaptiven Handlungskompetenz sich günstig auf die Förderung von Interesse und Selbstkonzept auswirken. Abbildung 4 stellt die Korrelationskoeffizienten dar zwischen den Ausprägungen der einzelnen Dimensionen der adaptiven Lehrkompetenz und der Veränderung des naturwissenschaftlichen Interesses und Fähigkeitsselbstkonzepts (hier gemessen als einfache Differenz zwischen Nachtest und Vortest). Effekte auf eine positive Entwicklung von Interesse und Selbstkonzept gehen hauptsächlich von den didaktischen und diagnostischen Kompetenzen der Lehrpersonen aus. Die Klassenführungskompetenzen zeigen weder einen Einfluss auf die Entwicklung des Interesses noch auf das Selbstkonzept. Als zusammenfassendes Mass wurde aus dem Mittelwert der drei Dimensionen eine Gesamtskala adaptive Handlungskompetenz gebildet. Diese korreliert signifikant mit der bereichsspezifischen Interessenentwicklung.

Zusätzliche Analysen zeigen, dass im Allgemeinen die Effekte in der Primarstufe stärker ausfallen als in der Sekundarstufe I (Beck et al., 2006). Die Planungskompetenzen korrelieren aber auch in der Primarstufe nicht signifikant mit den SRL-Merkmalen. 


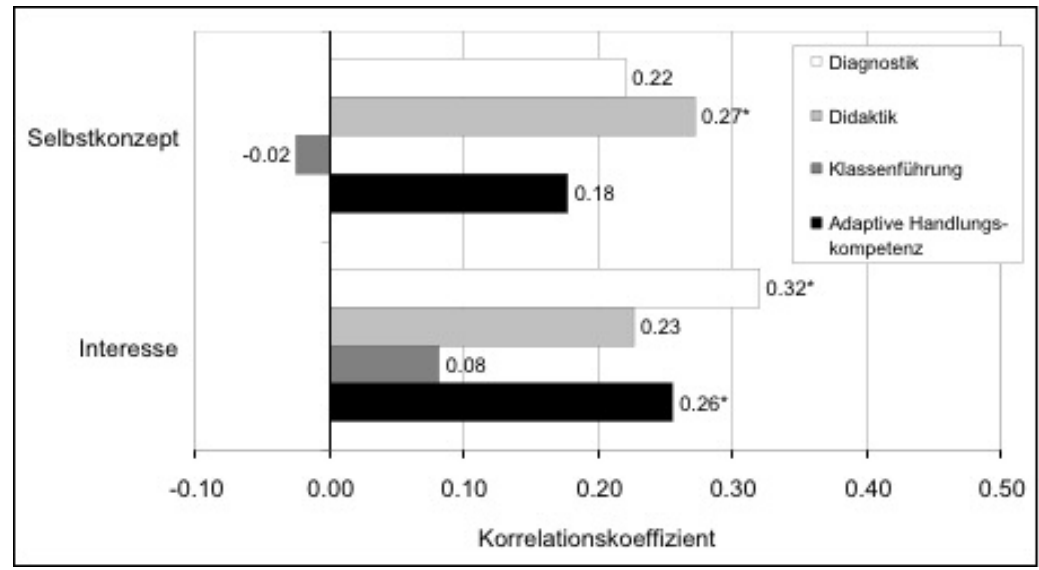

Anmerkung: $\mathrm{N}=47$ Lehrpersonen bzw. Klassen. ${ }^{*} \mathrm{p}<.05$.

Abbildung 4: Korrelationen zwischen den Dimensionen adaptiver Handlungskompetenz und der Veränderung des naturwissenschaftlichen Interesses und Selbstkonzepts zwischen Vor- und Nachtest.

Die hohe Bedeutung diagnostischer Kompetenzen von Lehrpersonen für die Entwicklung von bereichsspezifischem Interesse bestätigt sich auch, wenn ein anderes Mass für die Diagnosekompetenz herangezogen wird. So mussten die Lehrpersonen im Rahmen des Projekts "Adaptive Lehrkompetenz» nach einer selbst durchgeführten Unterrichtsreihe die Lernzielerreichung der einzelnen Schülerinnen und Schüler diagnostizieren. Als Mass für die Diagnosekompetenz dient die Korrelation zwischen der Testleistung der Schülerinnen und Schüler und der Leistungseinschätzung durch die Lehrpersonen (Genauigkeit des diagnostischen Urteils). Die bei der eigenen Klasse gemessene diagnostische Kompetenz der Lehrperson korreliert sowohl mit der im Videotest gemessenen Diagnosekompetenz $(\mathrm{r}=.47)$ als auch mit der Entwicklung des naturwissenschaftlichen Interesses während eines Schuljahrs $(r=.41)$. Diese Ergebnisse können als Hinweis auf die Validität der mittels Videotest gemessenen Diagnosekompetenz interpretiert werden.

\section{Diskussion und Folgerungen für die Schulpraxis}

Die vertieften Analysen der PISA-Daten konnten die hohe Bedeutung des SRL für die individuellen Leistungsergebnisse erhärten, insbesondere für das mathematische Selbstkonzept und Interesse. Erst wenn das Leistungsniveau der Schulen und der Schultyp konstant gehalten werden, wird der eigentliche $\mathrm{Zu}$ sammenhang zwischen individueller Leistung und Selbstkonzept ersichtlich. 
Auch wenn in den vorliegenden Analysen die Merkmale des SRL als abhängige Variablen modelliert wurden, ist einmal mehr anzumerken, dass mit den querschnittlich erhobenen PISA-Daten die kausale Richtung der Effekte nicht geprüft werden kann. Für die Zusammenhänge zwischen Selbstkonzept, Interesse und Leistung werden wechselseitige Effekte geltend gemacht. Entsprechend konnten Köller und Baumert (2001) in einer Längsschnittstudie belegen, dass das Begabungsselbstkonzept auch unter Konstanthaltung wichtiger konkurrierender Prädiktoren wie z.B. des Vorwissens oder der Leistungsstärke der Schulen im Verlauf von mehr als drei Jahren einen Effekt auf die Leistung aufweist. Daraus lässt sich folgern, dass nicht nur im Sinne des BFLPE die Leistung das Selbstkonzept beeinflusst, sondern umgekehrt auch dem Selbstkonzept eine eigenständige Wirkung auf die Leistung zukommt.

Mit der simultanen Modellierung von individueller Leistung, Schultyp und mittlerer Schulleistung konnte der BFLPE (Köller \& Baumert, 2001; Marsh, 1987) auch für die Schweiz repliziert werden. Schülerinnen und Schüler mit gleichen Mathematikkompetenzen verfügen in leistungsstärkeren Schulen und in anspruchsvolleren Schultypen über ein geringeres mathematisches Selbstkonzept. In geringerem Ausmass kann auch für das mathematische Interesse ein BFLPE nachgewiesen werden, wobei dieser hauptsächlich über das mathematische Selbstkonzept vermittelt wird. Der BFLPE kann mit sozialen Vergleichsprozessen innerhalb der eigenen Bezugsgruppe begründet werden. Schülerinnen und Schüler vermögen offenbar die eigenen Fähigkeiten nicht objektiv einzuschätzen, sondern neigen in leistungsstarken Lerngruppen dazu, die eigenen Fähigkeiten als geringer zu bewerten als Schülerinnen und Schüler mit gleichen individuellen Leistungen in einer leistungsschwächeren Lernumgebung. Um die psychosozialen Nachteile leistungsstarker Schulen zu verringern, ist für den Unterricht in anspruchsvollen Schultypen besonders wichtig, dass sich Leistungsbewertungen weniger an der sozialen Bezugsnorm ausrichten, sondern stärker an der Erreichung von Lernzielen (sachliche Bezugsnorm) und an individuellen Leistungsfortschritten orientieren. Köller Schnabel et Baumert (2000) weisen weiter darauf hin, dass die Art und Weise wie soziale Vergleiche in Schulklassen erzeugt werden, berücksichtigt werden müssen. Sie äussern Bedenken gegenüber einem aggressiv-kompetitiven Lernklima, in dem die Schülerinnen und Schüler als oberstes Ziel besser als ihre Kameraden abschneiden wollen. Als Folge davon treten psychosoziale Bereiche oder der Wunsch nach Kompetenzzuwachs in den Hintergrund.

Schon aus den ersten PISA-Analysen ist hervorgegangen, dass der selbstberichtete Einsatz von Lernstrategien weniger zur Erklärung von Mathematikkompetenzen beiträgt als das mathematische Selbstkonzept und das Interesse (OECD, 2004). Die vertieften Analysen zu den Lernstrategien haben dank der gemeinsamen Berücksichtigung des Schultyps und der Leistungsstärke von Schulen teilweise neue Erklärungsansätze geliefert. 
Bestätigen liess sich die negative Beziehung der Memorierstrategien zur individuellen Leistung. Memorierstrategien werden häufiger von Schülerinnen und Schülern mit schwächeren Leistungen gewählt. Strategien des Auswendiglernens werden in allen Schultypen gleich häufig eingesetzt und hängen nicht vom Leistungsniveau der Schule ab.

Der für die Schweiz gefundene negative Zusammenhang zwischen der individuellen Leistung und den Elaborationsstrategien (Brühwiler \& Biedermann, 2005) wandelt sich dagegen bei Konstanthaltung des Leistungsniveaus der Schulen und dem Schultyp in einen schwachen positiven Effekt. Der negative Zusammenhang bei einer einfachen Regressionsanalyse ist eine Folge davon, dass Elaborationsstrategien an leistungsstärkeren Schulen weniger häufig eingesetzt werden. Möglicherweise legen Lehrpersonen in Schultypen mit geringeren Ansprüchen beim Lernen grösseren Wert auf den Gebrauch solcher tiefenorientierter Lernstrategien.

Insgesamt sind die Beziehungen zwischen den Lernstrategien und den individuellen Mathematikleistungen als moderat zu bezeichnen. Dieser Befund darf indes nicht einfach dahingehend interpretiert werden, dass Lernstrategien für das schulische Lernen unbedeutend sind. Bei der Interpretation ist das Problem der situationsübergreifenden Erfassung von Lernstrategien mittels Selbsteinschätzungen zu bedenken. Fragebogenerhebungen unterschätzen in der Regel die Beziehung der Lernstrategien mit den fachlichen Leistungen. Artelt (2000) fordert deshalb eine situationsbezogene Erfassung der Lernstrategien. Leopold und Leutner (2004) betonen, dass die Qualität der eingesetzten Lernstrategien für den Lernertrag entscheidend sei. Dann liessen sich auch höhere und konsistentere Korrelationen zwischen dem Einsatz von Lernstrategien und der fachlichen Leistung finden.

Für die Schulpraxis sind Erkenntnisse zu den Fördermöglichkeiten des SRL, und was Lehrpersonen dazu beisteuern können, besonders relevant. Die Analysen zur Bedeutung handlungssteuernder Lehrerkognitionen haben gezeigt, dass sich eine hohe adaptive Handlungskompetenz günstig auf die Entwicklung von bereichsspezifischen Interessen und Selbstkonzepten der Schülerinnen und Schüler auswirkt. Dies gilt insbesondere für didaktische und diagnostische Kompetenzen, die sich unmittelbar auf das Unterrichtsgeschehen beziehen. Planungskompetenzen der Lehrpersonen spielen für die Förderung des SRL eine untergeordnete Rolle, haben sich jedoch für fachlichen Lernerfolg als zentral erwiesen (Beck et al., 2006).

Dieser Befund deutet darauf hin, dass für den Aufbau bereichsspezifischer Interessen und selbstbezogener Fähigkeitskognitionen die Lehrer-Schüler-Interaktion ausschlaggebend ist. Es scheint, dass es Lehrpersonen mit hoher adaptiver Handlungskompetenz besser gelingt, in konkreten Unterrichtssituationen motivierend auf individuelle Fragen oder Verständnisschwierigkeiten einzelner Schülerinnen und Schüler einzugehen oder Selbstwert stärkende Rückmeldungen zu 
geben. Ein hoher Stellenwert kommt dabei den diagnostischen Kompetenzen zu, die akkurate Einschätzungen der kognitiven Lernvoraussetzungen und affektiv-emotionalen Bedürfnisse der einzelnen Schülerinnen und Schüler erlauben.

Die Ergebnisse weisen in Übereinstimmung mit anderen Forschungsbefunden (z.B. Artelt, 2000; Zimmerman \& Martinez-Pons, 1990) darauf hin, dass mit zunehmendem Alter der Schülerinnen und Schüler die Ausprägung bereichsspezifischer Interessen und Selbstkonzepte abnehmen. Aus praktischer Sicht ist es von Interesse, wenn dieser negative Verlauf lernrelevanter Einstellungen durch das Handeln von Lehrpersonen mit hoher adaptiver Lehrkompetenz zumindest abzuschwächen wäre. Die praktische Relevanz betrifft nicht zuletzt die Lehrerinnen- und Lehrerbildung, da anzunehmen ist, dass sich Wissen und Kompetenzen von Lehrpersonen über Aus- und Weiterbildung verändern lassen.

Die gefundenen Effekte adaptiver Lehrkompetenzen zur Förderung gewisser Lernermerkmale sind insofern bemerkenswert, als eine erhebliche kausale Distanz zwischen dem Professionswissen von Lehrpersonen und dem Lernerfolg der Schülerinnen und Schüler besteht (Mandl \& Gerstenmaier, 2000). In diesem Zusammenhang sind die geplanten vertiefenden Analysen, welche die Beziehungen zwischen adaptiver Lehrkompetenz, Aspekten der Unterrichtsqualität und Lernleistungen der Schülerinnen und Schüler untersuchen, von besonderem Interesse. Dadurch soll empirisch belegt werden, wie sich adaptive Lehrkompetenz im unterrichtlichen Handeln manifestiert und vermittelt über Unterrichtsprozesse das schulische Lernen unterstützt. Selbstverständlich dürfen sich Massnahmen zur Förderung von SRL nicht allein auf Lehrerkognitionen fokussieren. Andere Ansätze wie beispielsweise gezieltes lernprozessorientiertes Strategietraining haben ebenfalls nachweislich zu besseren Lernergebnissen geführt (Leopold \& Leutner, 2004). Eine Kombination beider Ansätze dürfte besonders Erfolg versprechend sein.

\section{Anmerkungen}

1 Dieser Teil des Beitrags basiert auf dem vom Schweizerischen Nationalfonds (SNF) geförderten Projekt "Adaptive Lehrkompetenz - Analyse von Struktur, Veränderbarkeit und Wirkung handlungssteuernden Lehrerwissens» (SNF-Projekt Nr. 1114-066726.01). Gesuchsteller: Erwin Beck, Matthias Baer und Titus Guldimann; weitere Mitarbeitende: Sonja Bischoff, Christian Brühwiler, Peter Müller, Ruth Niedermann, Marion Rogalla und Franziska Vogt.

2 Die wörtlichen Itemformulierungen finden sich im ersten internationalen Bericht PISA 2003 (OECD, 2004).

3 Das Modell unter Einbezug des Selbstkonzepts ergibt einen Regressionskoeffizienten von $\beta=.72$ für das mathematische Selbstkonzept auf das Interesse. Mit Ausnahme des Schultyps Gymnasium ( $\beta=-.10$ ) erreicht kein weiterer Koeffizient einen absoluten Wert grösser als .06. Allerdings sind diese Ergebnisse äusserst vorsichtig zu interpretieren, da die sehr hohe Korrelation zwischen mathematischem Selbstkonzept und Interesse auf Multikollinearität hindeutet. Dadurch können Betagewichte und Teststatistiken verzerrt sein (z.B. Bortz, 1993). 
4 Vertiefende mehrebenenanalytische Auswertungen sind geplant.

5 Unter Annahme einer Irrtumswahrscheinlichkeit von $\alpha=.10$, da es sich um gerichtete Hypothesen handelt.

\section{Literatur}

Artelt, C. (2000). Strategisches Lernen. Münster: Waxmann.

Artelt, C., Baumert, J., Julius-McElvany, N. \& Peschar, J. (2003). Das Lernen lernen. Voraussetzungen für lebensbegleitendes Lernen. Ergebnisse von PISA 2000. Paris: OECD.

Artelt, C., Demmerich, A. \& Baumert, J. (2001). Selbstreguliertes Lernen. In J. Baumert, E. Klieme, M. Neubrand, M. Prenzel, U. Schiefele, W. Schneider, P. Stanat, K.-J. Tillmann \& M. Weiss (Hrsg.), PISA 2000. Basiskompetenzen von Schülerinnen und Schülern im internationalen Vergleich (S. 271-298). Opladen: Leske + Budrich.

Baumert, J., Köller, O. \& Schnabel, K.-U. (2000). Schulreformen als differentielle Entwicklungsmilieus - eine ungehörige Fragestellung? In M. Demmer (Hrsg.), Messung sozialer Motivation. Eine Kontroverse (S. 28-68). Frankfurt am Main: GEW.

Beck, E., Baer, M., Guldimann, T., Bischoff, S., Brühwiler, C., Niedermann, R., Müller, P., Rogalla, M. \& Vogt, F. (2006). Adaptive Lehrkompetenz. Analyse von Struktur, Veränderbarkeit und Wirkung handlungssteuernden Lehrerwissens. Wissenschaftlicher Schlussbericht, SNF Projekt Nr. 1114-066726.01. St.Gallen: Pädagogische Hochschule St.Gallen. (erscheint 2007 bei Waxmann)

Bischoff, S., Brühwiler, C. \& Baer, M. (2005). Videotest zur Erfassung «adaptiver Lehrkompetenz». Beiträge zur Lehrerbildung, 23 (3), 382-397.

Boekaerts, M. (1997). Self-regulated learning: A new concept embraced by researchers, policy makers, educators, teachers, and students. Learning and Instruction, 7 (2), 161-186.

Boekaerts, M. (1999). Self-regulated learning: Where we are today. International Journal of Educational Research, 31, 445-475.

Bortz, J. (1993). Statistik für Sozialwissenschaftler. Berlin: Springer.

Brühwiler, C. \& Biedermann, H. (2005). Selbstreguliertes Lernen als Voraussetzung für erfolgreiches Mathematiklernen. In C. Zahner Rossier (Hrsg.), PISA 2003: Kompetenzen für die Zukunft. Zweiter nationaler Bericht (S. 57-73). Neuchâtel: BFS/EDK.

Cohen, J. (1988). Statistical power analysis for the behavioral sciences. Hillsdale, NJ: Lawrence Erlbaum Associates.

Cronbach, L. J. \& Snow, R. E. (Hrsg.). (1977). Aptitudes and instructional methods. New York: Irvington/Naiburg.

Gruber, H., Mandl, H. \& Renkl, A. (2000). Was lernen wir in Schule und Hochschule: Träges Wissen? In H. Mandl \& J. Gerstenmaier (Hrsg.), Die Kluft zwischen Wissen und Handeln. Empirische und theoretische Lösungsansätze (S. 139-156). Göttingen: Hogrefe.

Gruehn, S. (2000). Unterricht und schulisches Lernen. Schüler als Quellen der Unterrichtsbeschreibung. Münster: Waxmann.

Helmke, A. (2003). Unterrichtsqualität: Erfassen, Bewerten, Verbessern. Seelze: Kallmeyersche Vertragsbuchhandlung.

Helmke, A. \& Weinert, F. E. (1997). Bedingungsfaktoren schulischer Leistungen. In F. E. Weinert (Hrsg.), Psychologie des Unterrichts und der Schule (S. 71-176). Göttingen: Hogrefe.

Jagacinski, C. M. \& Nicholls, J. G. (1990). Reducing effort to protect perceived ability: "they'd do it but I wouldn't." Journal of Educational Psychology, 82 (1), 15-21.

Köller, O. \& Baumert, J. (2001). Leistungsgruppierungen in der Sekundarstufe I. Ihre Konsequenzen für die Mathematikleistung und das mathematische Selbstkonzept der Begabung. Zeitschrift für Pädagogische Psychologie, 15 (2), 99-110.

Köller, O. \& Schiefele, U. (2003). Editorial zum Themenschwerpunkt selbstreguliertes Lernen im Kontext von Schule und Hochschule. Zeitschrift für Pädagogische Psychologie, 17 (3/4), 155-177. 
Köller, O., Schnabel, K. U. \& Baumert, J. (2000). Der Einfluss der Leistungsstärke von Schulen auf das fachspezifische Selbstkonzept der Begabung und das Interesse. Zeitschrift für Entwicklungspsychologie und Pädagogische Psychologie, 32 (2), 70-80.

Leopold, C. \& Leutner, D. (2002). Der Einsatz von Lernstrategien in einer konkreten Lernsituation bei Schülern unterschiedlicher Jahrgangsstufen. Zeitschrift für Pädagogik, 45. Beiheft, 240-258.

Leopold, C. \& Leutner, D. (2004). Selbstreguliertes Lernen und seine Förderung durch prozessorientiertes Training. In J. Doll \& M. Prenzel (Hrsg.), Bildungsqualität von Schule. Lehrerprofessionalisierung, Unterrichtsentwicklung und Schülerförderung als Strategien der Qualitätsverbesserung (S. 364-376). Münster: Waxmann.

Lüdtke, O., Köller, O., Bundt, S., Gomolka, J. \& Waterman, R. (2004). Durchführung und methodische Grundlagen der TOSCA-Studie. In O. Köller, R. Waterman, U. Trautwein \& O. Lüdtke (Hrsg.), Wege zur Hochschulreife in Baden-Württemberg. TOSCA - Eine Untersuchung an allgemein bildenden und beruflichen Gymnasien (S. 121-151). Opladen: Leske + Budrich.

Mandl, H. \& Gerstenmaier, J. (2000). Die Kluft zwischen Wissen und Handeln. Empirische und theoretische Lösungsansätze. Göttingen: Hogrefe.

Marsh, H. W. (1987). The big-fish-little-pond effect on academic self-concept. Journal of Educational Psychology, 79 (3), 280-295.

Marsh, H. W. (1990). Influences of internal and external frames of reference on the formation of math and English self-concepts. Journal of Educational Psychology, 82 (1), 107-116.

Mason, J. (1994). Linking qualitative and quantitative data analysis. In A. Bryman \& R. G. Burgess (Hrsg.), Analyzing qualitative data (S. 89-110). London: Routledge.

Mislevy, R. J., Johnson, E. G. \& Muraki, E. (1992). Scaling procedures in NAEP. Journal of Educational Statistics, 17, 131-154.

Moser, U. \& Berweger, S. (2005). Soziale Herkunft und Mathematikleistung: Ein vertiefter Blick auf die Kantone. In C. Zahner Rossier (Hrsg.), PISA 2003: Kompetenzen für die Zukunft. Zweiter nationaler Bericht (S. 99-118). Neuchâtel: BFS/EDK.

OECD (2001). Lernen für das Leben. Erste Ergebnisse von PISA 2000. Paris: OECD.

OECD (2004). Lernen für die Welt von morgen. Erste Ergebnisse von PISA 2003. Paris: OECD.

OECD (2005). PISA 2003 data analysis manual. Paris: OECD.

Ramseier, E. \& Brühwiler, C. (2003). Herkunft, Leistung und Bildungschancen im gegliederten Bildungssystem: Vertiefte PISA-Analyse unter Einbezug der kognitiven Grundfähigkeiten. Schweizerische Zeitschrift für Bildungswissenschaften, 25 (1), 23-58.

Raudenbush, S., Bryk, A., Cheong, Y. F., Congdon, R. \& du Toit, M. (2004). HLM 6. Hierarchical linear and nonlinear modeling. Lincolnwood: Scientific Software International, Inc.

Schiefele, U. \& Schreyer, I. (1994). Intrinsische Lernmotivation und Lernen. Ein Überblick zu Ergebnissen der Forschung. Zeitschrift für Pädagogische Psychologie, 8 (1), 1-13.

Schunk, D. H. (1990). Introduction to the special section on motivation and efficacy. Journal of Educational Psychology, 82 (1).

Shute, V. \& Towle, B. (2003). Adaptive e-learning. Educational Psychologist, 38 (2), 105-114.

Wang, M. C. (1980). Adaptive instruction: Building on diversity. Theory into Practice, 19 (2), 122-128.

Weinert, F. E. (1996). Lerntheorien und Instruktionsmodelle. In F. E. Weinert (Hrsg.), Psychologie des Lernens und der Instruktion. Enzyklopädie der Psychologie, Pädagogische Psychologie (S. 1-48). Göttingen: Hogrefe.

Zahner Rossier, C. (Hrsg.). (2005). PISA 2003: Kompetenzen für die Zukunft. Zweiter nationaler Bericht. Neuchâtel: BFS/EDK.

Zimmerman, B. J. \& Martinez-Pons, M. (1990). Student differences in self-regulated learning: Relating grade, sex, and giftedness to self-efficacy and strategy use. Journal of Educational Psychology, 82 (1), 51-59. 
Zutavern, M. \& Brühwiler, C. (2002). Selbstreguliertes Lernen als fächerübergreifende Kompetenz. In BFS/EDK (Hrsg.), Für das Leben gerüstet? Die Grundkompetenzen der Jugendlichen - Nationaler Bericht der Erhebung PISA 2000 (S. 64-89). Neuchâtel: BFS/EDK.

Schlagworte: adaptive Lehrkompetenz, selbstreguliertes Lernen, Wirksamkeit von Lehrpersonen, Fischteich-Effekt, Mathematikleistung, diagnostische Kompetenz, didaktische Kompetenz, Interesse, Selbstkonzept

\section{L'impact des contextes scolaires et des compétences d'un enseignement adaptatif pour un apprentissage autodirigé}

\section{Résumé}

Etre capable de réguler son activité d'apprentissage est une compétence essentielle en tant quadulte en charge de sa formation continue. L'apprentissage autorégulé (AAR) doit être compris comme étant à la fois une condition et un objectif d'apprentissage à l'école. A partir des données PISA 2003, la présente contribution analyse, dans un premier temps, les effets des types d'écoles et du niveau de performances des écoles sur différents aspects de l'AAR. Les résultats montrent que le "big-fish-little-pond-effect" (BFLPE; Marsh, 1987) se produit aussi dans les écoles suisses. À compétences égales en mathématiques, les élèves fréquentant des écoles dites performantes se perçoivent moins compétents en mathématiques que leurs pairs fréquentant des écoles dites peu performantes.

Etant donné l'importance de l'AAR dans la réussite scolaire, une deuxième étude examine dans quelle mesure les enseignants contribuent à favoriser l'apprentissage autorégulé. Les données proviennent des 49 enseignant-es et de leurs classes qui ont participé à la recherche du FNRS sur les «Compétences dun enseignement adapté». Cette notion désigne la capacité des enseignants à adapter la préparation et la gestion de l'enseignement aux différents niveaux des élèves en classe, et de mettre en place les conditions favorables à l'apprentissage. Les résultats montrent que les compétences diagnostiques et didactiques des enseignants sont primordiales pour renforcer l'intérêt et le sentiment de compétence des élèves dans chaque discipline scolaire, pour autant qu'elles se manifestent directement dans l'activité effective d'enseignement.

Mots clés: compétence d'enseignement adapté, apprentissage autorégulé, efficacité d'enseignement, big-fish-little-pond-effect, compétences en mathématiques, compétences diagnostiques, compétences didactiques, intérêt, concept de soi 


\title{
L'impatto del contesto scolastico e delle competenze didattiche adattive sull'apprendimento autoregolato
}

\author{
Riassunto \\ Per sviluppare un'attitudine all'apprendimento autonomo al termine del per- \\ corso scolastico, è necessario imparare a regolare con successo i propri processi di \\ apprendimento.
}

L'apprendimento autoregolato (AAR) è da considerarsi nel contempo oggetto e condizione dell'apprendimento scolastico. Partendo dai dati PISA 2003, il presente contributo analizza dapprima l'impatto del tipo di scuola e del livello di prestazioni delle scuole su diversi aspetti dell'AAR. I risultati dello studio mostrano per la Svizzera il fenomeno "big-fish-little-pond-effect» (BFLPE; Marsh, 1987), dove le allieve e gli allievi delle scuole più performanti dichiarano un'immagine di sé in matematica inferiore a quella rilevata presso allieve e allievi che attestano prestazioni individuali di medesimo livello rispetto ai primi, ma che frequentano scuole con prestazioni inferiori.

Considerato il forte impatto dell'AAR sul successo scolastico, ci si è pure chinati sui contributi che gli insegnanti possono fornire per promuovere l'apprendimento autoregolato. La base empirica per le analisi effettuate riguarda un campione di 49 insegnanti e le loro classi, soggetti dello studio del Fondo Nazionale Svizzero sulle «competenze didattiche adattive». Con il termine di competenze didattiche adattive si intende la capacità dei docenti di progettare e realizzare dei corsi adattandoli alle situazioni individuali delle persone in formazione, creando delle condizioni favorevoli alla comprensione di ciò che viene appreso. I risultati dimostrano che per favorire l'interesse per le singole discipline e sviluppare l'immagine di sé, le competenze diagnostiche e didattiche degli insegnanti sono determinanti nella misura in cui queste vengono applicate direttamente ai corsi.

Parole chiave: competenze didattiche adattive, apprendimento autoregolato, efficacia dell'insegnamento, big-fish-little-pond-effect, competenze in matematica, competenze diagnostiche, competenze didattiche, interesse, immagine di sé

\section{Effects of school context and adaptive teaching competency on self-regulated learning}

\section{Summary}

The ability to regulate one's own learning process is of high importance for adult self-directed learning. Self-regulated learning (SRL) needs to be understood as both, a condition as well as a goal of learning in school contexts. This contribution examines the results of PISA in 2003 analyses of the effects of type of school and school achievement on self-regulated learning. The investigation reveals that 
the «big-fish-little-pond-effect» (BFLPE; Marsh, 1987) occurs in Swiss schools too. Students with the same level of competency in mathematics have a lower mathematical self concept when attending a school with good results compared to students at a less successful school. Considering the substantial influence of self-regulated learning on learning outcomes, a second study examines the contribution of teachers to foster self regulated learning. 49 teachers and their students took part in "Adaptive Teaching Competency», a research project which was supported by the Swiss National Science Foundation. Adaptive teaching competency is defined as the teacher's ability to adjust lesson planning as well as teaching performance to the diverse abilities of students in class, thus achieving optimal conditions for comprehensive learning. The results indicate that the competence of teachers in the dimensions of diagnosis and teaching methods is vital to the promotion of domain specific interest and self concept. Adaptive teaching competency is closely linked to the context of the classroom.

Keywords: adaptive teaching competency, self-regulated learning, teacher effectiveness, big-fish-little-pond-effect, mathematics achievement, diagnosis of students' learning, teaching methods, interest, self concept 
T h e m a 